\section{Frühkindliche Entwicklung auf den Punkt gebracht}

- Professor Manfred Cierpka ist auch Autor des umfassenden Handbuches „Frühe Kindheit 0-3 Jahre", das ebenfalls im Springer-Verlag erschienen ist. Dort werden viele Grundlagen noch ausführlicher dargestellt, sowohl was die körperliche und seelische Entwicklung des Kindes angeht als auch damit zusammenhängende mögliche Veränderungen und Belastungen in Partnerschaft und Familie. Außerdem werden darin Konzepte der Prävention, die im Bereich der "Frühen Hilfen" eine immer größere Rolle spielen, aufgeführt.

Im Buch "Regulationsstörungen“ wurden die klinischen Kapitel aus „Frühe Kindheit 0-3 Jahre" für die Praxis aufbereitet und die Problematik der Regulationsstörungen anhand von Kasuistiken sinnvoll ergänzt und auf den Punkt gebracht. Dieses kompaktere und vor allem stärker praxiskonzentrierte Buch behandelt in insgesamt elf Kapiteln die wichtigsten Störungen der frühen Ver- haltensregulation, das heißt Schrei-, Schlafund Fütterstörungen. Im Weiteren folgen unter anderem „Ausführungen zum exzessiv anklammernden Verhalten", "Persistierendes Trotzen und aggressives Verhalten" sowie Erläuterungen zur "Spielunlust" (gemeint sind damit Regulationsprobleme von Aufmerksamkeit und Spielen).

Jedes Kapitel zeigt recht schlüssig auf, wie sich Ansätze zu einem therapeutischen Vorgehen finden lassen. Ziel ist es immer, eine "Passung zwischen Eltern und Kind (wieder-)herzustellen" und eine gute Qualität der Eltern-Kind-Beziehung zu erarbeiten. Finden sich länger dauernde, komplexere oder schwerwiegendere Beeinträchtigungen der elterlichen Fähigkeiten, dann besteht eine Indikation für eine Psychotherapie. Insgesamt wird hier ein abgestuftes Vorgehen vorgestellt, das sich ähnlich auch in den Leitlinien der Deutschen Gesellschaft für Sozialpädiatrie wiederfindet. Außerdem betont das vorliegende Werk den richtigen Hinweis, Regulationsstörungen beim Kind nicht vorschnell zu pathologisieren, andererseits Tendenzen rechtzeitig zu erkennen, die Eltern und ihre Kinder überlasten.

Das Buch wendet sich an alle mit frühkindlichen Entwicklungen befassten Berufsgruppen und stellt so für Kinderärzte, Kinderkrankenschwestern und medizinische Fachangestellte wie auch für Hebammen ein gut verständliches, am Arbeitsalltag orientiertes und insgesamt sehr lesenswertes Buch dar. Der Preis ist bei dieser hohen Informationsdichte und guten Ausstattung mehr als angemessen. Dr. Ulrich Mutschler

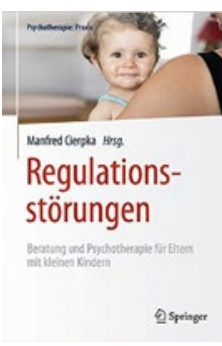

Manfred Cierpka (Hrsg.)

Regulationsstörungen Springer Verlag, Berlin 2015 ISBN 978-3-642-40741-3 $29,99 €$

\title{
Ehrliche Beschreibung eines Tabuthemas
}

_ Der Autor reflektiert in dem vorliegenden Buch sein Leben mit der bipolaren Depression: von den beeinträchtigenden Veränderungen in seinem Alltag bis hin zum Quasistillstand und dann nach Selbsteinweisung in eine psychiatrische Klinik, nach Diagnosestellung und adäquater Therapie das Wiederaufleben, Abrutschen in eine manische Phase und schließlich langsam „wieder im Alltag ankommen“. Eindrücklich schildert er gerade in der manischen Phase seine „Denkflüsse“ mit immer kürzer werdenden Sätzen, sodass der Leser selbst außer Atem gerät und das Getriebene, Rastlose erlebt. Er setzt sich kritisch mit dem Umgang mit Depressionen in der Öffentlichkeit auseinander und beschreibt seine eigenen Strategien, um mit seiner Depression, aber auch der Manie leben zu können und die Kontrolle zu behalten.

Das Buch ist so packend und gleichzeitig auch mit viel Humor geschrieben (was ja schon der Titel hoffen lässt), dass ich die 200 Seiten ohne Unterbrechung gelesen habe - trotz eines erschöpfenden Arbeitstages.
Der Leser lernt nicht nur viel über die Geschichte der Depression, er erlebt durch die gute Selbstreflektion und Eigenbeschreibung die depressiven und vor allem manischen Phasen hautnah mit. Er nimmt aus der Sicht des Betroffenen den Klinikalltag wahr, von den Pflegern über das ärztliche Personal bis zu den "Mitbewohnern".

Bemerkenswert finde ich auch seine Einstellung zur Depression: ein „banales hirnchemisches Schicksal" und davon ausgehend die Akzeptanz der medikamentösen Therapie. Diese bringt keineswegs die vollständige Heilung, aber eine so deutliche Besserung, dass wieder Lebensqualität besteht. Kritisch hinterfragt der Autor die in der Gesellschaft und Laienpresse immer wieder propagierte Pharmaverteufelung und ideologisch vertretene "Suche im tiefsten Inneren nach einer unglücklichen Kindheit als Ursache der Depression". Leider betrifft dies nicht nur Depressionen, sondern auch viele andere neurobiologische Störungen. Bis in die 1980er-Jahre wurde Autismus noch auf die "Kühlschrankmutter" zurück- geführt. Bei ADHS wird eine Bindungsstörung noch heute diskutiert, wenn es überhaupt als Störung akzeptiert wird.

Das Buch ist uneingeschränkt für alle zu empfehlen - nicht nur für Ärzte -, um einen besseren Zugang zu dem Erleben ihrer Patienten zu erhalten oder zu ihrer eigenen Depression. Auch Nichtbetroffene profitieren von dem ehrlichen Umgang mit den dargestellten Problemen und mit der Auseinandersetzung von "nature" und "nurture". Es ist zu hoffen, dass die Lektüre des Buches hilft, das Tabu Depression zu beseitigen und den Betroffenen die gesellschaftliche Integration zu ermöglichen.

Dr. Kirsten Stollhoff

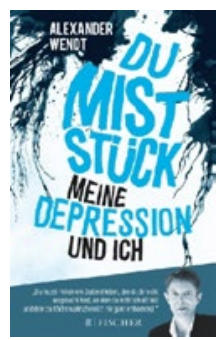

Alexander Wendt

Du Miststück - Meine Depression und ich

S. Fischer Verlag, Frankfurt/Main 2016 ISBN 978-3-596-03539-7 $16,99 €$ 